Proc. of the 15th Int. Workshop on Slow Positron Beam Techniques and Applications, Prague, September 2-6, 2019

\title{
J-PET Monte Carlo Simulations for Time-Reversal Symmetry Test in Ortho-Positronium Decay
}

\author{
J. RAJ*, D. KisieleWska AND E. CZERWińsKi \\ ON BEHALF OF THE J-PET COLlaboration \\ Jagiellonian University, Krakow, Poland
}

\begin{abstract}
This article reports the development of Monte Carlo simulations of the J-PET detector using the Geant4 toolkit aimed at serving as a foundation for testing the time-reversal symmetry in the decay of ortho-Positronium (o-Ps) atoms. In order to observe asymmetries in time-reversal, it is important to understand the physical properties of the signal candidates (o-Ps $\rightarrow 3 \gamma$ ) recorded by the detector setup. The simulations aim at replicating the experimental procedure of producing meta-stable triplet states of positronium and testing the T-symmetry using symmetry-odd operators with the Jagiellonian-Positron Emission Tomograph (J-PET) detector.
\end{abstract}

DOI: 10.12693/APhysPolA.137.137

PACS/topics: 11.30.Er, 36.10.Dr, 13.40.Hq

\section{Introduction}

Discrete symmetries and their conservations have played a fundamental role in exploring particle physics. Classical physics has taught us the demand for conservation laws to be invariant under reversal of time. The demonstration of the mirror symmetry being violated by the weak interaction was the first discovery in this domain [1]. So far, there has not been any experimental evidence of a violation of these discrete symmetries in the charged leptonic sector [2-5]. The Jagiellonian Positron Emission Tomograph (J-PET) is the first PET scanner making use of plastic scintillators $[6,7]$, which is capable of positronium imaging $[8,9]$ as well as testing discrete symmetries in the decay of positronium atoms [10]. This experiment aims at producing the triplet state of a positronium to test the symmetry by applying the time-reversal operator [10]. As a foundation to these studies, this article describes J-PET-Geant4, which is a program designed to run Monte Carlo simulations of the J-PET detector created using the Geant4 toolkit.

\section{Time-Reversal Symmetry}

Time reversal symmetry violations have not been observed in purely leptonic systems, so far [2-5]. The Standard Model predicts photon-photon or weak interaction to both give rise to phenomena mimicking a violation of the symmetry in the order of $10^{-9}$ (photonphoton interaction) and $10^{-13}$ (weak interactions), respectively $[3,4,11-13]$. There is a difference of about 6 orders of magnitude between the present experimental upper limit and the standard model predictions $[5,14,15]$. Discrete symmetries were proposed

*corresponding author; e-mail: juhi.raj@doctoral.uj.edu.pl
TABLE I

Discrete symmetry odd-operator constructed using the momentum direction of the primary annihilation photons $\left(\boldsymbol{k}_{i}\right)$ and the secondary scattered annihilation photons $\left(\boldsymbol{k}_{i}^{\prime}\right)$ from the decay of o-Ps. The vector $\left(\boldsymbol{\epsilon}_{i}\right)$ is constructed as the cross product of the momentum direction of the primary photon $\left(\boldsymbol{k}_{i}\right)$ and its corresponding secondary scattered photon $\left(\boldsymbol{k}_{i}^{\prime}\right)$. The descending momentum of the three annihilation photons are denoted by $\left|\boldsymbol{k}_{i}\right|>\left|\boldsymbol{k}_{j}\right|>\left|\boldsymbol{k}_{k}\right|$.

\begin{tabular}{c|c|c|c|c|c}
\hline \hline Operator & $\mathrm{C}$ & $\mathrm{P}$ & $\mathrm{T}$ & $\mathrm{CP}$ & CPT \\
\hline $\boldsymbol{\epsilon}_{i} \cdot \boldsymbol{k}_{j}$ & + & - & - & - & +
\end{tabular}

to be tested with the ortho-Positronium (o-Ps) system by determining the expectation value of the symmetry odd-operator [10]. The symmetry-odd operator $\boldsymbol{\epsilon}_{i} \cdot \boldsymbol{k}_{j}$ is constructed using the most-probable linear polarization vector:

$$
\boldsymbol{\epsilon}_{i}=\boldsymbol{k}_{i} \times \boldsymbol{k}_{i}^{\prime}
$$

where $\boldsymbol{k}_{i}$ is the momentum directions of the primary annihilation photon and $\boldsymbol{k}_{i}^{\prime}$ is the momentum direction of its corresponding secondary scattered annihilation photon. The observation of a non-zero expectation value of this operator would imply non-invariance of a given symmetry. Symmetries for which the given operator is odd can be tested at the J-PET system. This concerns, namely, $\mathrm{P}, \mathrm{T}$, and CP symmetries (marked " - ") as showcased in Table I [10].

\section{Monte-Carlo Simulation}

The J-PET detector consists of 192 plastic scintillator strips $500 \times 19 \times 7 \mathrm{~mm}^{3}$ in size, as shown in Fig. 1a [16]. Those strips form three concentric layers, i.e., 48 modules arranged as a cylinder with a radius of $425 \mathrm{~mm}, 48$ modules arranged as a cylinder with a radius $467.5 \mathrm{~mm}$, and 96 modules as a cylinder with radius $575 \mathrm{~mm}$. Only the most probable decay of o-Ps into 


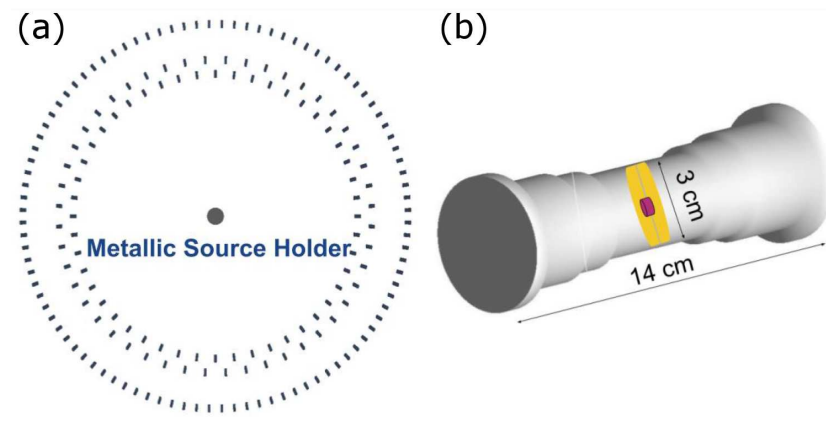

Fig. 1. (a) Transverse view of the simulated J-PET detector with a metallic chamber placed in the center of the detector geometry.(b) Side view of the metallic chamber (grey cylindrical tube) holding a $\beta^{+}$source (red) in the center with XAD-4 porous polymer covering the source (yellow).
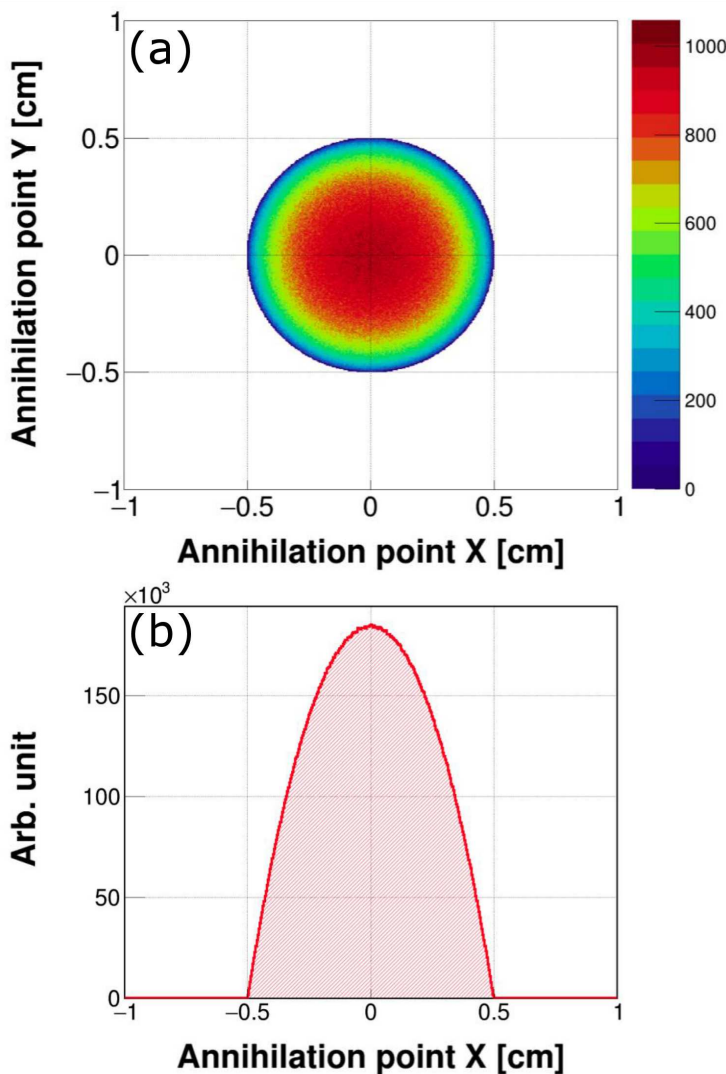

Fig. 2. (a): The spectrum shows the distribution of annihilation positions of the produced signal candidates using the J-PET-Geant4 simulations. (b) Projection of the spectrum on the left-panel.

three photons $\left(\mathrm{BR}(\mathrm{o}-\mathrm{Ps} \rightarrow 4 \gamma / \mathrm{o}-\mathrm{Ps} \rightarrow 3 \gamma)<2.6 \times 10^{-6}\right.$ at $90 \%$ C.L. $[3,10])$ is implemented in the J-PET-Geant 4 simulator [17]. Since the aim of this research is testing T-symmetry at the sensitivity level of $10^{-4}$, the less frequent decays of o-Ps are neglected. These annihilation gamma quanta are required to be co-planar in the Center


Fig. 3. (a) Distribution of angles between the registered annihilation photons from the decay of o-Ps $\rightarrow 3 \gamma$.

(b) Energy spectrum of annihilation photons from o-Ps $\rightarrow 3 \gamma$ events.

of Mass (CM) frame in order to conserve momentum [18]. The $\beta^{+}$source is placed in a metallic chamber covered in XAD-4 porous polymer in order to aid the production of ortho-Positronium atoms [16]. A geometrical model (as shown in Fig. 1) replicating the experimental setup was employed to set up the Monte Carlo simulations. The distribution of annihilation positions of the generated signal events are shown in Fig. 2.

The generation of signal candidates is validated by observing the distribution of the relative angles between the three registered annihilation photons as shown in Fig. 3a, and the distribution of the photon energies of the registered signals in the J-PET detector as shown in Fig. 3b.

\section{Conclusion}

The production of the J-PET Monte Carlo simulations using the Geant 4 toolkit lays a foundation for testing the time-reversal symmetry in the decay of orthoPositronium atoms. The Monte Carlo simulation program designed for the J-PET detector created in Geant-4 is an open-source code [20]. The simulation program is versatile and applicable to various experiments performed with the J-PET detector. The J-PET simulation 
program is compatible with the J-PET framework software, which can, therefore, be used to reconstruct Monte Carlo data with exactly the same procedure as for actual experimental data. Hence, a direct comparison of measurements and simulations is allowed [21].

In order to experimentally observe violation of the time-reversal symmetry, it is important to simulate signal events to predict their behaviour in absence of any violation. The simulations were performed to replicate the experimental procedure of producing ortho-Positronium atoms. The simulation also tests the T-symmetry using one of the symmetry-odd operator with the simulated Jagiellonian-Positron Emission Tomograph (J-PET) detector.

\section{Acknowledgments}

This work was supported by the National Science Center of Poland, through the OPUS 11 grant No. 2016/21/B/ST2/01222 and the Jagiellonian University DSC grant No. 2019-N17/MNS/000036.

\section{References}

[1] J. Christenson, J.W. Cronin, V.L. Fitch, R. Turlay, Phys. Rev. Lett. 13, 138 (1964).

[2] M.S. Sozzi, Discrete Symmetries and CP Violation, Oxford University Press (2008).

[3] S.D. Bass, Acta Phys. Pol. B 50, 1319, (2019).

[4] K. Abe et al., (T2K Collaboration), Phys. Rev. Lett. 121, 171802 (2018).

[5] T. Yamazaki, T. Namba, S. Asai, T. Kobayashi, Phys. Rev. Lett. 104, 083401 (2010).
[6] P. Moskal, Sz. Niedźwiecki, T. Bednarski, et al., Nucl. Instr. Meth. A 764, 317 (2014).

[7] P. Moskal, O. Rundel, D. Alfs, et al. Phys. Med. Biol. 61, 2025 (2016)

[8] P. Moskal, D. Kisielewska, C. Curceanu, et al., Phys. Med. Biol. 64, 055017 (2019).

[9] P. Moskal, B. Jasińska, E.Ł. Stępień, S.D. Bass, et al., Nature Revs. Phys. 1, 527 (2019).

[10] P. Moskal, D. Alfs, T. Bednarski, et al., Acta Phys. Pol. B 47, 509 (2016).

[11] W. Bernreuther, U. Löw, J.P. Ma, O. Nachtmann, Z. Phys. C 41, 143 (1988)

[12] B.K. Arbic, S. Hatamian, M. Skalsey, J. Van House, W. Zheng,, Phys. Rev. A 37, 3189 (1988).

[13] A. Pokraka, A. Czarnecki, Phys. Rev. D 96, 093002 (2017).

[14] V.A. Kostelecky, N. Russell, Rev. Mod. Phys. 83, 11 (2011).

[15] P.A. Vetter, S.J. Freedman, Phys. Rev. Lett. 91 , 263401 (2003).

[16] S. Niedźwiecki, P. Białas, C. Curceanu, et al., Acta Phys. Pol. B 48, 1567 (2017).

[17] D. Kamińska, Ph.D. Thesis, Jagiellonian University, 2018.

[18] D. Kamińska, A. Gajos, E. Czerwiński, et al., Eur. Phys. J. C 76, 445 (2016).

[19] B. Jasińska, M. Gorgol, M. Wiertel, et al., Acta Phys. Pol. B 47, 453 (2016).

[20] D. Kisielewska, S. Sharma, K. Kacprzak, J. Chhokar, K. Rakoczy, J-PET-Geant4, 2019.

[21] W. Krzemień, D. Alfs, P. Białas, et al., Acta Phys. Pol. B 47, 561 (2016). 